\title{
Erratum: Improved Monte Carlo Glauber predictions at present and future nuclear colliders [Phys. Rev. C 97, 054910 (2018)]
}

\author{
Constantin Loizides, Jason Kamin, and David d'Enterria
}

(Received 19 October 2018; published 31 January 2019)

DOI: 10.1103/PhysRevC.99.019901

We correct an error made in the calculation of the overlap area, shown in the top panels of Fig. 16 and reported in Tables XI-XXI, of the original article. In the original article, we report the overlap area, defined in Eq. (5), for various systems. It is computed in the Monte Carlo Glauber approach by evaluating the area of wounded nucleons with two fine-grained grids, one for participants from each nucleus as explained in Appendix B of Ref. [1]. This approach avoids approximations using participant position distributions and ad hoc normalizations. However, we made an error by taking the "AND" rather than the "OR" of the two fine-grained grids. The former computes the true geometrical intersect of the participants but not the total area the participant distributions actually cover. The corrected figure is shown in Fig. 1, whereas the numerical values are given in Tables I-III.

The overall conclusions of the original article are not affected.
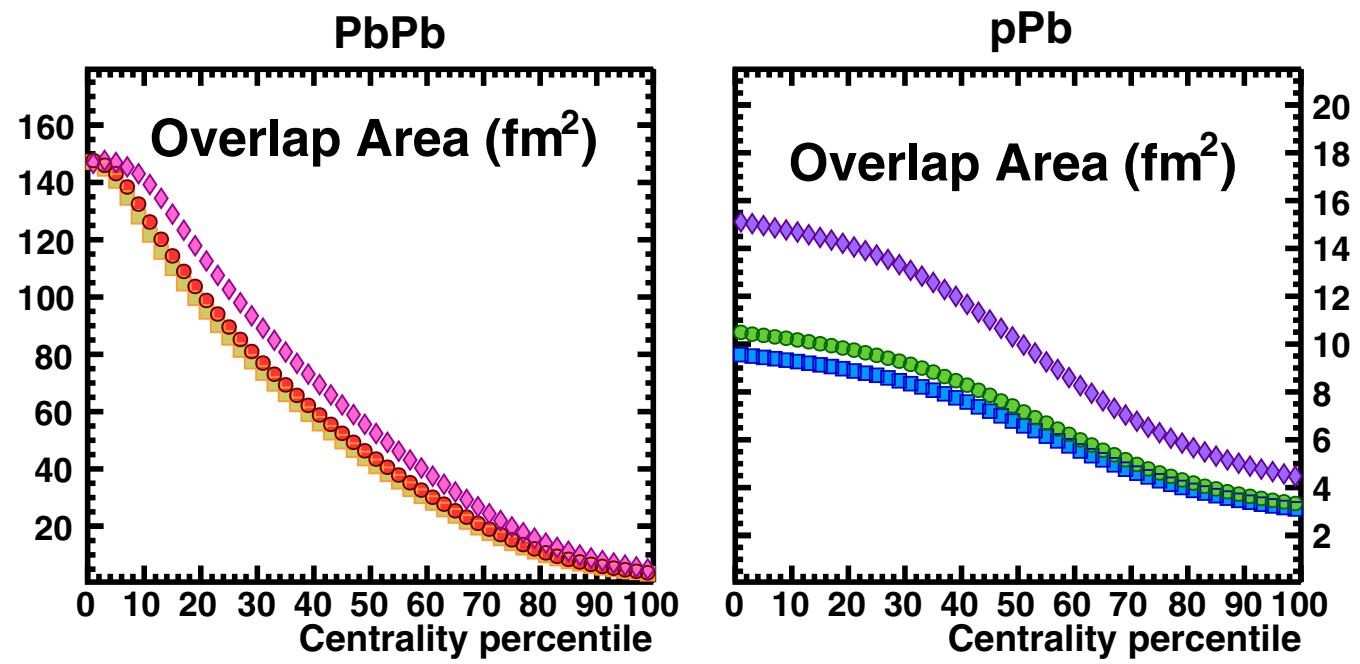

FIG. 1. Overlap area $\left(A_{\mathrm{T}}\right)$ as a function of centrality for $\mathrm{PbPb}$ collisions at $\sqrt{s_{\mathrm{NN}}}=2.76,5.02$, and 39 TeV (bottom to top curves in the left panel) and for $p \mathrm{~Pb}$ collisions at $\sqrt{s_{\mathrm{NN}}}=5.02,8.16$ and $63 \mathrm{TeV}$ (bottom to top curves in the right panel). The two panels replace the two top panels of Fig. 16 in the original paper.

Published by the American Physical Society under the terms of the Creative Commons Attribution 4.0 International license. Further distribution of this work must maintain attribution to the author(s) and the published article's title, journal citation, and DOI. 
TABLE I. Mean overlap area $A_{\perp}$ and standard deviation in $\mathrm{fm}^{2}$ for $\mathrm{PbPb}$ collisions. Replaces corresponding columns in Tables IX-XIII.

\begin{tabular}{|c|c|c|c|c|c|}
\hline Centrality & $\mathrm{PbPb}, 2.76 \mathrm{TeV}$ & $\mathrm{PbPb}, 5.02 \mathrm{TeV}$ & $\mathrm{PbPb}, 5.5 \mathrm{TeV}$ & $\mathrm{PbPb}, 10.6 \mathrm{TeV}$ & $\mathrm{PbPb}, 39 \mathrm{TeV}$ \\
\hline $0-5 \%$ & $144 \pm 4.67$ & $145 \pm 3.9$ & $145 \pm 3.81$ & $146 \pm 3.17$ & $147 \pm 2.31$ \\
\hline $5-10 \%$ & $133 \pm 7.96$ & $136 \pm 7.48$ & $137 \pm 7.38$ & $140 \pm 6.55$ & $144 \pm 4.79$ \\
\hline $15-20 \%$ & $103 \pm 7.89$ & $108 \pm 8.19$ & $108 \pm 8.21$ & $113 \pm 8.53$ & $122 \pm 9$ \\
\hline $20-25 \%$ & $91.3 \pm 7.65$ & $95.3 \pm 7.95$ & $95.8 \pm 8.03$ & $100 \pm 8.33$ & $109 \pm 8.91$ \\
\hline $35-40 \%$ & $61.7 \pm 7.02$ & $64.8 \pm 7.34$ & $65.3 \pm 7.39$ & $68.8 \pm 7.72$ & $76 \pm 8.38$ \\
\hline $40-45 \%$ & $53.4 \pm 6.85$ & $56.4 \pm 7.15$ & $56.8 \pm 7.22$ & $60 \pm 7.55$ & $66.7 \pm 8.24$ \\
\hline $45-50 \%$ & $45.8 \pm 6.68$ & $48.6 \pm 6.99$ & $48.9 \pm 7.06$ & $51.9 \pm 7.41$ & $58.1 \pm 8.07$ \\
\hline $50-55 \%$ & $38.8 \pm 6.49$ & $41.3 \pm 6.84$ & $41.6 \pm 6.89$ & $44.4 \pm 7.23$ & $50 \pm 7.93$ \\
\hline $55-60 \%$ & $32.3 \pm 6.32$ & $34.6 \pm 6.67$ & $34.9 \pm 6.7$ & $37.3 \pm 7.1$ & $42.5 \pm 7.82$ \\
\hline $75-80 \%$ & $12.1 \pm 5.09$ & $13.2 \pm 5.48$ & $13.4 \pm 5.52$ & $14.6 \pm 5.96$ & $17.3 \pm 6.84$ \\
\hline $80-85 \%$ & $8.98 \pm 4.33$ & $9.81 \pm 4.69$ & $9.92 \pm 4.73$ & $10.9 \pm 5.13$ & $13 \pm 5.99$ \\
\hline $85-90 \%$ & $6.67 \pm 3.45$ & $7.32 \pm 3.76$ & $7.4 \pm 3.8$ & $8.14 \pm 4.14$ & $9.76 \pm 4.87$ \\
\hline $90-95 \%$ & $5.02 \pm 2.53$ & $5.51 \pm 2.76$ & $5.56 \pm 2.78$ & $6.14 \pm 3.05$ & $7.39 \pm 3.62$ \\
\hline $95-100 \%$ & $3.76 \pm 1.54$ & $4.13 \pm 1.69$ & $4.19 \pm 1.72$ & $4.62 \pm 1.89$ & $5.6 \pm 2.27$ \\
\hline $0-100 \%$ & $50.7 \pm 41.7$ & $51.9 \pm 41.9$ & $52.1 \pm 41.9$ & $53.4 \pm 42$ & $55.9 \pm 42.2$ \\
\hline
\end{tabular}

TABLE II. Mean overlap area $A_{\perp}$ and standard deviation in $\mathrm{fm}^{2}$ for $p \mathrm{~Pb}$ collisions. Replaces corresponding columns in Tables XIV-XVIII.

\begin{tabular}{|c|c|c|c|c|c|}
\hline $0-5 \%$ & $9.51 \pm 1.2$ & $10.4 \pm 1.24$ & $10.6 \pm 1.25$ & $12 \pm 1.3$ & $15 \pm 1.38$ \\
\hline $5-10 \%$ & $9.37 \pm 1.22$ & $10.3 \pm 1.27$ & $10.4 \pm 1.27$ & $11.8 \pm 1.33$ & $14.8 \pm 1.43$ \\
\hline $10-15 \%$ & $9.22 \pm 1.24$ & $10.1 \pm 1.29$ & $10.3 \pm 1.29$ & $11.6 \pm 1.36$ & $14.6 \pm 1.47$ \\
\hline $15-20 \%$ & $9.04 \pm 1.27$ & $9.91 \pm 1.33$ & $10 \pm 1.33$ & $11.4 \pm 1.4$ & $14.3 \pm 1.52$ \\
\hline $20-25 \%$ & $8.81 \pm 1.3$ & $9.67 \pm 1.36$ & $9.8 \pm 1.37$ & $11.1 \pm 1.44$ & $13.9 \pm 1.58$ \\
\hline $30-35 \%$ & $8.25 \pm 1.37$ & $9.03 \pm 1.44$ & $9.16 \pm 1.46$ & $10.3 \pm 1.55$ & $12.9 \pm 1.73$ \\
\hline $35-40 \%$ & $7.88 \pm 1.41$ & $8.61 \pm 1.49$ & $8.72 \pm 1.5$ & $9.81 \pm 1.6$ & $12.2 \pm 1.8$ \\
\hline $40-45 \%$ & $7.43 \pm 1.45$ & $8.2 \pm 1.52$ & $8.23 \pm 1.53$ & $9.23 \pm 1.65$ & $11.4 \pm 1.88$ \\
\hline $45-50 \%$ & $6.95 \pm 1.46$ & $7.58 \pm 1.54$ & $7.68 \pm 1.56$ & $8.58 \pm 1.67$ & $10.6 \pm 1.92$ \\
\hline $50-55 \%$ & $6.43 \pm 1.46$ & $6.99 \pm 1.55$ & $7.07 \pm 1.56$ & $7.91 \pm 1.68$ & $9.7 \pm 1.93$ \\
\hline $75-80 \%$ & $4.11 \pm 1.1$ & $4.43 \pm 1.18$ & $4.49 \pm 1.19$ & $4.96 \pm 1.3$ & $6.02 \pm 1.53$ \\
\hline $80-85 \%$ & $3.8 \pm 0.964$ & $4.1 \pm 1.04$ & $4.14 \pm 1.04$ & $4.57 \pm 1.14$ & $5.53 \pm 1.36$ \\
\hline $85-90 \%$ & $3.54 \pm 0.814$ & $3.81 \pm 0.869$ & $3.85 \pm 0.884$ & $4.25 \pm 0.967$ & $5.53 \pm 1.14$ \\
\hline $90-95 \%$ & $3.33 \pm 0.649$ & $3.57 \pm 0.688$ & $3.61 \pm 0.695$ & $3.99 \pm 0.767$ & $4.81 \pm 0.898$ \\
\hline $95-100 \%$ & $3.15 \pm 0.451$ & $3.39 \pm 0.483$ & $3.43 \pm 0.487$ & $3.78 \pm 0.533$ & $4.55 \pm 0.626$ \\
\hline $0-100 \%$ & $6.5 \pm 2.53$ & $7.09 \pm 2.78$ & $7.18 \pm 2.82$ & $8.06 \pm 3.19$ & $999 \pm 4.02$ \\
\hline
\end{tabular}


TABLE III. Mean overlap area $A_{\perp}$ and standard deviation in $\mathrm{fm}^{2}$ for XeXe collisions at $\sqrt{s_{\mathrm{NN}}}=5.44 \mathrm{TeV}$ as well as $\mathrm{AuAu}$ and $\mathrm{CuCu}$ collisions at $\sqrt{s_{\mathrm{NN}}}=0.2 \mathrm{TeV}$. Replaces corresponding columns in Tables XIX-XXI.

\begin{tabular}{|c|c|c|c|}
\hline Centrality & $\mathrm{XeXe}, 5.44 \mathrm{TeV}$ & $\mathrm{AuAu}, 0.2 \mathrm{TeV}$ & $\mathrm{CuCu}, 0.2 \mathrm{TeV}$ \\
\hline $0-5 \%$ & $111 \pm 6.89$ & $123 \pm 7.49$ & $51.8 \pm 4.11$ \\
\hline $5-10 \%$ & $99.5 \pm 7.5$ & $106 \pm 7.22$ & $45.5 \pm 4.18$ \\
\hline $15-20 \%$ & $78.7 \pm 7.63$ & $80.8 \pm 6.65$ & $35 \pm 4.12$ \\
\hline $20-25 \%$ & $69.8 \pm 7.59$ & $70.6 \pm 6.43$ & $30.6 \pm 4.08$ \\
\hline $35-40 \%$ & $47.4 \pm 7.36$ & $45.8 \pm 5.95$ & $19.7 \pm 3.96$ \\
\hline $40-45 \%$ & $41.1 \pm 7.28$ & $39.1 \pm 5.79$ & $16.8 \pm 3.9$ \\
\hline $45-50 \%$ & $35.2 \pm 7.2$ & $33 \pm 5.66$ & $14.1 \pm 3.82$ \\
\hline $50-55 \%$ & $29.8 \pm 7.09$ & $27.4 \pm 5.49$ & $11.8 \pm 3.71$ \\
\hline $55-60 \%$ & $24.8 \pm 6.98$ & $22.3 \pm 5.29$ & $9.78 \pm 3.54$ \\
\hline $75-80 \%$ & $10.2 \pm 5.12$ & $7.95 \pm 3.75$ & $4.52 \pm 2.31$ \\
\hline $80-85 \%$ & $8.11 \pm 4.3$ & $5.92 \pm 3.09$ & $3.78 \pm 1.93$ \\
\hline $85-90 \%$ & $6.47 \pm 3.46$ & $4.43 \pm 2.42$ & $3.18 \pm 1.57$ \\
\hline $90-95 \%$ & $5.18 \pm 2.58$ & $3.34 \pm 1.75$ & $2.69 \pm 1.19$ \\
\hline $95-100 \%$ & $4.06 \pm 1.63$ & $2.48 \pm 1.04$ & $2.26 \pm 0.774$ \\
\hline $0-100 \%$ & $41.2 \pm 33.5$ & $41.1 \pm 36.5$ & $18.1 \pm 15.4$ \\
\hline
\end{tabular}

[1] C. Loizides, Glauber modeling of high-energy nuclear collisions at the subnucleon level, Phys. Rev. C 94, 024914 (2016). 